\title{
CDGSH Iron-Sulfur Domain-Containing Protein 1
}

National Cancer Institute

\section{Source}

National Cancer Institute. CDGSH Iron-Sulfur Domain-Containing Protein 1. NCI

Thesaurus. Code C113135.

CDGSH iron-sulfur domain-containing protein 1 (108 aa, $12 \mathrm{kDa}$ ) is encoded by the human CISD1 gene. This protein is involved in both iron-sulfur cluster binding and the regulation of cell respiration. 